\title{
Liquid sheet thickness measurements using multi-pass, time-gated femtosecond imaging
}

\author{
Dilip Sanadi ${ }^{1}$, Said Idlahcen ${ }^{1}$, Jean-Bernard Blaisot ${ }^{* 1}$,Fabien Thiesset ${ }^{1}$ \\ ${ }^{1}$ CORIA UMR-6614, Normandie Universite, UNIROUEN, CNRS, INSA,76000 Rouen, France \\ ${ }^{*}$ Corresponding author email: blaisot@coria.fr
}

\begin{abstract}
The present work focuses on the development and application of a non-invasive technique for measuring the thickness of a flat liquid sheet. The technique consists in separating a 100 femtosecond (fs) laser pulse into an imaging pulse which passes through the liquid sheet and a gating pulse, that travels only in air and whose path length can be adjusted using a delay line. The time delay $\Delta \tau$ between the imaging and gating pulse is directly proportional to the liquid sheet thickness $h$ and can be measured using Second Harmonic Generation (SHG) based time gate (here a Beta Barium Borate crystal is used for SHG). In order to enhance the thickness measurement resolution, an original multi-pass configuration was designed where the imaging pulse is passing twice (or more if needed) through the medium which increases the time delay between imaging and gating pulse. As a first step, we have checked the reliability of this technique by conducting measurements for a glass plate of known thickness $\left(\mathrm{h}_{g}=120 \mu \mathrm{m}\right)$. The measured thickness value $(117 \mu \mathrm{m})$ is in close agreement with the expected value. Then, attention has been paid on flat liquid sheets produced by single-hole fan spray nozzles with various water-glycerol solutions. The streamwise evolution of the measured thickness of the sheet exhibits good agreement with the semi-analytical model of Dombrowski et al. [1].
\end{abstract}

\section{Keywords}

Stability of sheets, Non-linear optics, Femtosecond imaging

\section{Introduction}

Inferring the liquid sheet thickness is primordial for understanding the complex multi-scale physics of the liquid-sheet disintegration and droplet formation. In particular, perforation holes appear on the sheet and extend up to the disintegration of the whole sheet to form ligaments and droplets. The growth rate of perforation holes has strong dependence on local liquid sheet thickness and thermo-physical properties of test fluid. Furthermore, with knowledge of liquid sheet thickness one can deduce the sheet shape theoretically and can compare with experimental stable and un-stable liquid sheet rims [2]. Consequently, for the engineering system design, there exists significant need to have a knowledge of liquid sheet thickness and of the stable and unstable regions in the liquid sheet. The most common optical techniques utilized for liquid sheet thickness characterization are spectral/spatial interferometry $[1,3]$. The main constraint with the interferometic techniques is its limited potential for spatial resolution of fringes and spectral resolution of the spectrometer which determines the limit of the thickness measurement. With the recent advancement in optical diagnostic tools, researchers have developed new techniques such as Terahertz time-domain spectroscopy (TDS) [4] or Infrared spectromicroscopy [5]. One of the major limitations of $\mathrm{THz}$ imaging is due to the high absorption rate of polar liquids, especially water. The absorption coefficient of water is as high as $150 \mathrm{~cm}^{-1}$ at 1 $\mathrm{THz}$. This property limits the sensing and imaging capabilities through variable thickness liquid sheet.

When a liquid is back-illuminated, the light undergoes optical deviation (refraction, reflection and diffraction) and intensity variations before reaching the detector. Specifically, optical distortions and intensity attenuation can arise when light passes through objects of differing refractive indices(Rl's). The optical path difference between a ray of light passing through a thickness $h$ of liquid and a ray passing through the same distance in the surrounding medium is characterized 
by a time delay $\Delta \tau=h \Delta n / c$, where, $\Delta n$ is the difference between the refractive index of liquid and surrounding medium and $c$ is the speed of light in vacuum. The time delay $\Delta \tau$ could be detected by selection of the light photons based on the their arrival time at the detector. Time gating (time-based filtering) allows selection of optical signal within short temporal window and can be applied to separate the light that is delayed due to multiple interactions with the object. This temporal window of time gate must be very short ( $\sim 10$ 's ps) in order to capture the useful signal. Non-linear optical processes then allows us to detect such a sub-picoseconds gating mechanisms including, Second Harmonic Generation [6,7] and Optical Kerr effect (OKE) $[8,9]$. Our method relies on the same working principle of Idlahcen et. al [6] with its applicability for liquid sheets. The aim of the present paper is to study the potential of SHG crystal time gate to extract the local liquid sheet thickness and subsequently study the application and development of this technique. The liquid sheet thickness is measured experimentally and compared with the semi-analytical sub-model of Dombrowwski et.al [1].

\section{Material and methods}

Before proceeding, we first carried out an exploratory qualitative characterization of various stable and unstable regimes of liquid sheet using a system of conventional backlight illumination. These backlight images were further used for measuring the liquid sheet angle which is required in the semi-analytical sub-model of Dombrowwski et.al [1].

The experimental test facility is shown in Figure 1 (a). This standard assembly comprises two main sections: (1) a central pipe fitted with a flat fan spray atomizer with an elliptical shape orifice installed in an optically accessible chamber. Two different nozzles of orifice hydraulic diameters $D_{h 1}=1.1 \mathrm{~mm}$ and $D_{h 2}=0.625 \mathrm{~mm}$ have been used. (2) a Storage vessel (volume capacity 10 liters) to pressurize the liquid with the compress air (gauge pressure 2 bar). Across all experiments various water-glycerol solutions ( $0-40 \%$ by volume) were used as a working fluid with dynamic viscosity $(\mu)$ ranging from 1-3.78 $\mathrm{mPa}$.s. The liquid flow rate was measured and calibrated for each mixture by using an inline flow rotameter (range $0.1-1.21 . \mathrm{min}^{-1}$ ). Physical and optical properties of the blends are summarized in Table. 1.

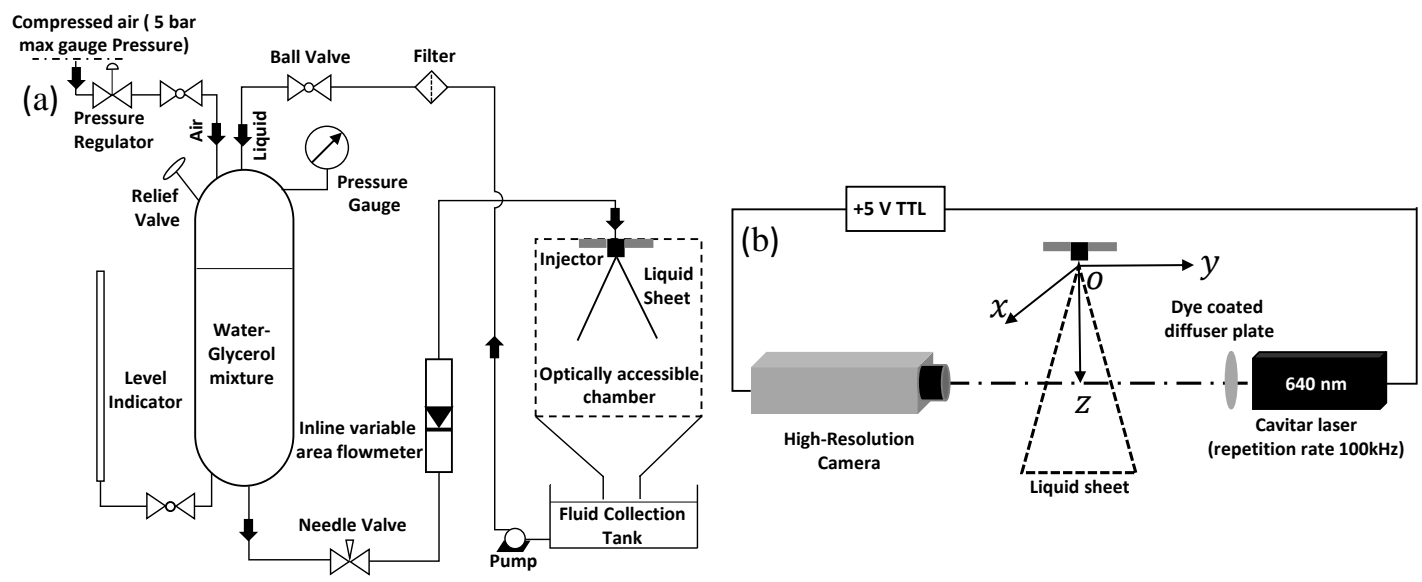

Figure 1. (a)Schematic illustration of experimental test facility (b)Schematic of Back-lit illumination for visualization of liquid sheet.

Conventional backlight illumination technique was employed to study the different topological structures of the liquid sheet with the goal of appraising the various stable and unstable regimes of such flow in the nozzle near-field region. Figure $1 \mathrm{~b}$ illustrates the arrangement of the backlight imaging system, consisting of a $200 \mathrm{~W}$ non-coherent laser diode source (Cavitar CAVILUX $\mathrm{HF}$ ) with tunable pulse duration. The light source has a wavelength $(\lambda)$ of $640 \mathrm{~nm}$ and maximum pulse rate of $100 \mathrm{kHz}$. We use a dye coated diffuser plate to ensure a uniform illumination density. Images were acquired by high resolution CCD camera (mvBlueCougar x125aG Matrix vision) with a pixel resolution of $2448 \times 2050$ pixels. The camera is coupled with a zoom lens $(f 16-100 \mathrm{~mm})$ a spatial field of view of $77 \times 87 \mathrm{~mm}^{2}$ (optical magnification of 0.080 ). 


\begin{tabular}{llllll}
\hline Liquid blend & $\mu(\mathrm{mPa.s})$ & $\nu\left(\mathrm{mm}^{2} \mathrm{~s}^{-}\right)$ & $\rho\left(\mathrm{kgm}^{-} 3\right)$ & $\sigma\left(\mathrm{mNm} \mathrm{m}^{-}\right)$ & $n$ \\
\hline$F_{0}$ & 1 & 1.0068 & 998.1 & 71.64 & 1.33 \\
$F_{10}$ & 1.28 & 1.2463 & 1012.4 & 71.07 & 1.34 \\
$F_{20}$ & 1.90 & 1.878 & 1038.2 & 70.86 & 1.35 \\
$F_{40}$ & 4.78 & 4.324 & 1100 & 70.04 & 1.38 \\
\hline
\end{tabular}

Table 1. The thermo-physical and optical properties of various water-glycerol aqueous mixture at $20^{\circ} \mathrm{C}, 1 \mathrm{~atm}$. (subscript 0 to 40 indicates percentage by volume of glycerol in water)

\section{Characterization of flow regimes}

The present section aims at identifying different stable and unstable flow regimes when the flow rate is progressively increased. This allows us to identify the most suited flow regimes for the liquid sheet thickness measurement to be measured with the femtosecond imaging technique. Figure 2(a)-2(e) shows the flow patterns observed when both Reynolds numbers $\left(\operatorname{Re}_{j}\right)$ and Weber numbers $\left(\mathrm{We}_{j}\right)$ are increased in the range of 450-12000 and 125-8000 respectively. $\mathrm{Re}_{j}$ and $\mathrm{We}_{j}$ are computed based on bulk velocity and nozzle hydraulic diameter. The different flow

Increase in We and Re for different aqueous solution of water glycerol
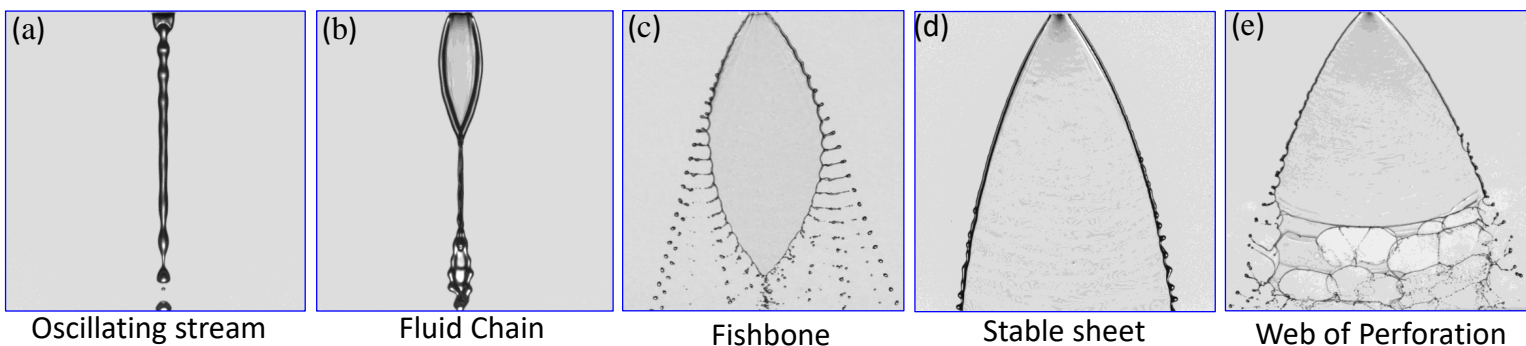

Figure 2. Distinct flow pattern produced by Flat fan atomizer for various solutions of water-glycerol

structures of a liquid-sheet flow are retrieved such as oscillating stream, fluid chain, fishbone structures, stable sheet and web of perforation. These all flow structures are consistent with previous work of impinging jet $[2,10,11]$. Here, we have made the choice of measuring the liquid-sheet thickness in the Stable regime (Figure 2(d)). The latter was found in the range $\mathrm{Re}_{j}=2000-6000$ and $\mathrm{We}_{j}=1500-6500$.

\section{Optical setup:Multi-pass time-gated femtosecond imaging}

A schematic of the optical setup for multi-pass, time-resolved femtosecond imaging with the SHG-based time-gate is shown in Figure 3(a). The laser system (Ti:Sapphire Regenerative amplifier, Coherent Inc.) generates 100 femto-second ( $f s)$ pulses $(800 \mathrm{~nm}, 1 \mathrm{kHz}, 3 \mathrm{~mJ})$. A combination of a rotatable half wave-plate $(\lambda / 2)$ and a linear polarizer $(P)$ is used to control the average power of the pulse. The incoming pulse is divided into imaging (probe) and gating (pump) pulses by means of a 50/50 Beam Splitter $\left(B_{1}\right)$. An Imaging pulse which crosses the liquid sheet and gating pulse is used to activate the gate for a very short duration. The computer operated delay stage is used to instigate a small delay between the imaging and gating pulses as well as to synchronize them to reach at the BBO crystal and produce Second Harmonic Generation (SHG: Frequency doubled) effect along an angle $\theta$. The spatio temporal superposition of two incoming pulses in the crystal generates third pulse in the intermediate direction at a wavelength of $400 \mathrm{~nm}$ (blue pulse). SHG is a nonlinear optical process with second order susceptibility $\chi^{(2)}$ in which two photons with the same frequency interact with a nonlinear material, are "combined" and generate a new photon with twice the frequency of 
the initial photons. In other words, it is a special case of sum-frequency generation where the wavelength of the SHG signal is exactly half of the wavelength of the incident primary beam, as shown in Figure3(b).

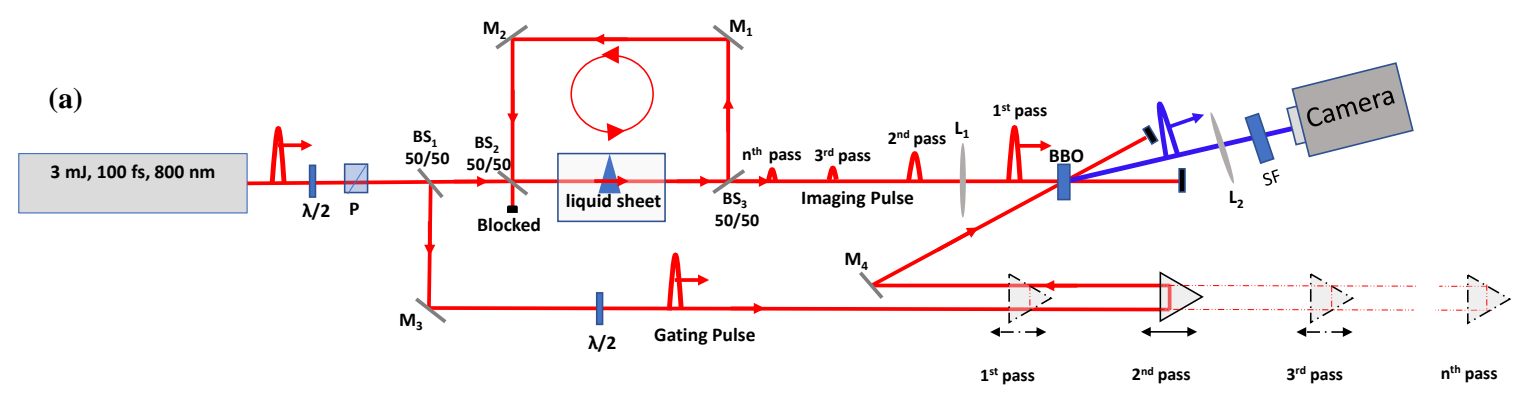

(b)
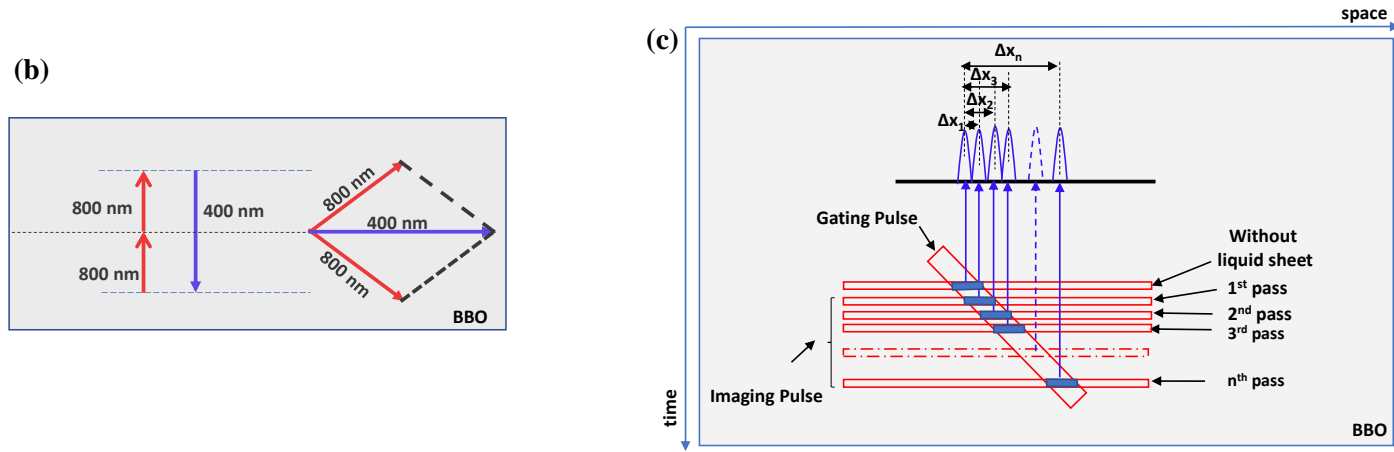

Figure 3. (a)Schematic of the experimental setup for multi-pass, the SHG based time-gate femtosecond imaging.Nomenclature: (BS) - 50 : 50 beam splitter, $\left(\mathrm{M}_{1}, \mathrm{M}_{2}, \mathrm{M}_{3}, \mathrm{M}_{4}\right)$ - mirrors, $(\lambda / 2)$-half waveplates, $(P)$-Linear Polarizer, (BBO) - $\beta$-barium borate crystal, $\left(L_{1}, L_{2}\right)$ - lenses.(b) Schematic of Second Harmonic Generation in BBO (non-linear Crystal)(c) Schematic showing the increase in spatial shift and temporal delay for multi-pass as $\Delta x_{2}, \Delta x_{3}$ and $\Delta x_{n}$.

The imaging beam from $\mathrm{BS}_{1}$ again passes through the beam splitter $\left(\mathrm{BS}_{2}\right)$ resulting in two separate optical paths, in which $50 \%$ of partially reflected beam passes through liquid sheet and $50 \%$ is blocked. The forward delayed light signal due to interaction with liquid sheet is then again partially reflected by $\mathrm{BS}_{3}$ and made to pass two times (more if needed) through liquid sheet with use of two mirrors $\left(M_{1}\right.$ and $\left.M_{2}\right)$ by forming cavity around the optical chamber. The idea of multi-pass is just to increase time delay by a factor 2 between imaging and gating pulse and hence increase the time resolution by a factor 2 (Figure 3(c)). By adjusting delay stage of gating pulse, one can set as many passes as needed and hence adjust the time resolution to the object to be qualified. This multiply passed, time delayed imaging beam is then collected using wide aperture biconvex lens $L_{1}$. The focal length of the lens can be chosen to get desired magnification of the object on CCD camera. Finally the imaging beam crosses the gating beam inside the BBO crystal to generate SHG beam. An additional neutral density filter is used to adjust the intensity of the light reaching the camera as per the requirement. The imaging system consist of Dantec FlowSense camera at a maximum frame rate of $44 \mathrm{fps}$ with pixel resolution of $1600 \times 1200$ (pixel size $=7.4 \mu \mathrm{m}$ ). The pulse duration is synchronized with camera shutter speed through TTL signal. A spatial band pass filter (SF) is used to removed undesired light.

\section{Working principal of a SHG gate}

If the gating pulse is incident at the BBO crystal at the same time as imaging pulse arrives, the optical time-gate is said to be in an open state (marked by point A and B in Figure 4a). When these two pulses overlap in SHG medium, one has to characterize spatio-temporal features of the light arriving on camera by changing the delay between the gating pulse and the imaging pulse. These two pulses crosses somewhere inside the crystal at an angle $\theta_{n}$ and produces third pulse at half angle. This intersection (marked by point $A$ in Figure 4a) produces bright intensity zone on the camera which is related to the cross-correlation of the imaging and gating beam maximum profiles. Next, with the stepwise increase in path length (Gating pulse 2 :Figure 
4a) of gating pulse by adjusting delay stage, the intersection point $A$ shifts to point $B$ and corresponding shift of bright zone $\Delta x$ appears on camera. Here at the point $\mathrm{B}$, the cross-correlation leads to a smaller intensity due to the fact that the beam maximum profiles never coincide in this case. Considering the triangular geometry of intersection points $A B C$ in Figure 4a leads to
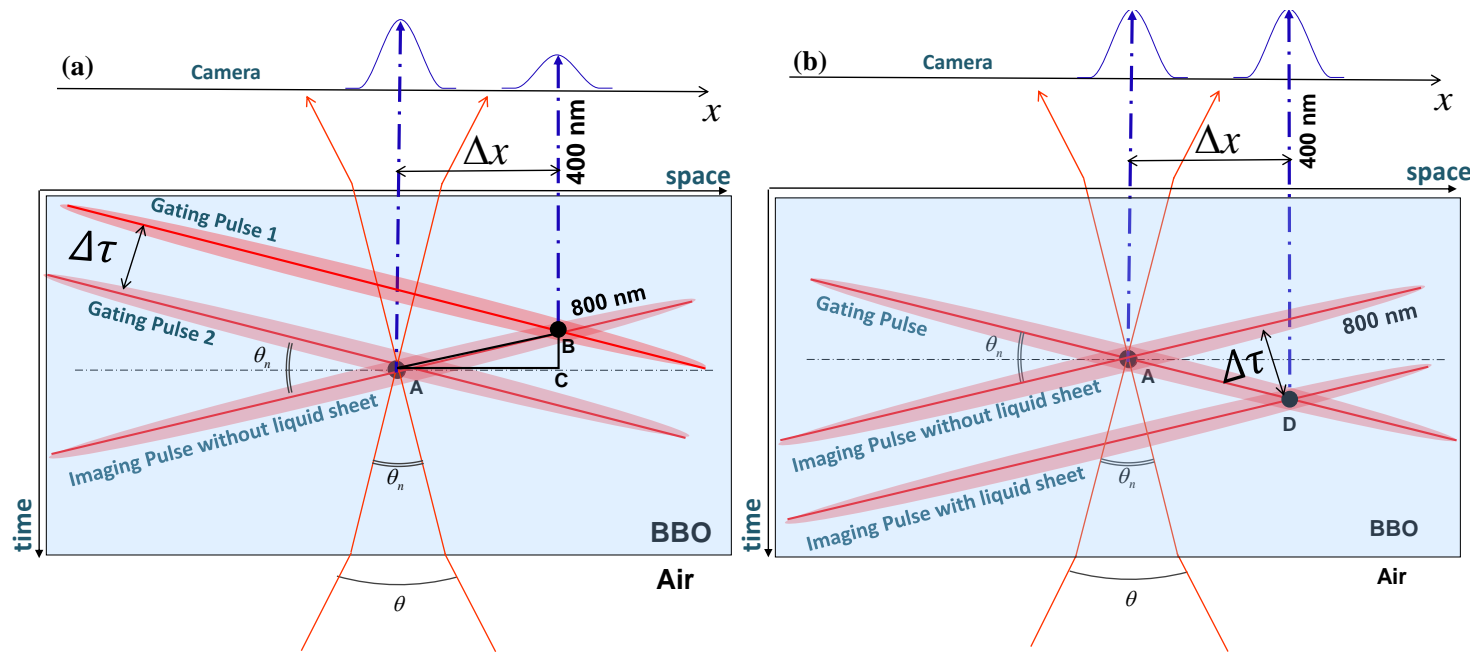

Figure 4. (a)Relation between gating pulses and imaging pulse passing (a) Air (without any object).(b) object under study. On the image, a pixel located at $\Delta x$ corresponds to a time delays with respect to the delayed photons.

relation between $\Delta \tau$ and $\Delta x$ as $\Delta \tau=2 \frac{\Delta x \sin \theta_{n} / 2}{c / n}$, where, $\theta_{n}$ is the angle between the two beams inside the crystal and $n$ the refractive index of BBO crystal. Now, if imaging pulse travels across a liquid sheet, a temporal delay of the image beam will occur due to transmission of light through higher refractive index medium than air. Figure 4(b) shows geometrical configuration corresponding to an overlap of the gating pulse without liquid sheet and with liquid sheet.The imaging pulse passed through the liquid sheet crosses gating pulse at point $D$ (see Figure $4 b$ ). On the camera, pixel shift occurs at distance $\Delta x$ along the horizontal axis from the known position of the directly transmitted photons gives an information on point $\mathrm{D}$ in terms of position $x$ and delay $\Delta \tau$. This implies that the intensity distribution $\mathrm{I}(\Delta \tau, x, y)$ is function of only horizontal axis position $x$ and delay $\Delta \tau$.

\section{Results and discussion:Spatio-Temporal diagrams}

The general intensity field $\hat{I}(x, y, t)$ of SHG beam as an output signal produced by pump and probe can be expressed in three dimensions as a purely spatial and temporal approach:

$$
\hat{I}(x, y, t)=\frac{c \epsilon_{0}}{2} \hat{A}(x, y, t)^{2}
$$

where, $\hat{A}(x, y, t)$ is its amplitude, $c$ is velocity of the light, and $\epsilon_{0}$ is vacuum permittivity. Considering this three dimensional information of intensity as an output signal one can study the interaction of the light and object under study. Fixed values of delay between gating pulse and imaging pulse, i.e. $\Delta \tau$ are considered, and for each, 10 images are recorded to produce a mean image $I(\Delta \tau, x, y)$ for a particular delay. These recorded average image for each individual delay contains the spatial and temporal information of intensity in 3D data cube $I(\Delta \tau, x, y)$ as shown in Figure 5(b). As discussed in previous section, the temporal dependence along the horizontal axis of the camera is taken into account. Then, Intensity $I(\Delta \tau, x)$ is obtained by averaging the pixels of each column $y$ of the images. The representative spatio-temporal Figure $5(d)$ is composed of directly transmitted photons without any object interaction, since light is passing through only air medium. Figure $5(\mathrm{e})$ corresponds to imaging pulse traveling through the centreline of the liquid sheet.

Quantitative analysis is done to estimate an average time delay due to interaction of light and liquid sheet. To find the location of local maximum intensities $I_{\max }(\Delta \tau, x, y)$ for various delays, 
(a)

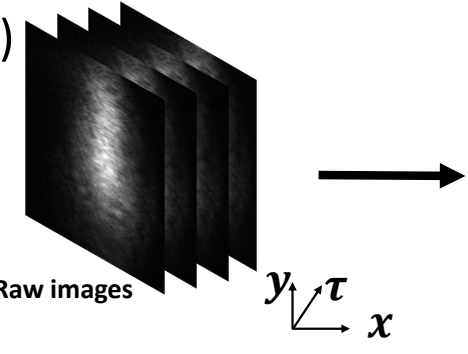

(b)

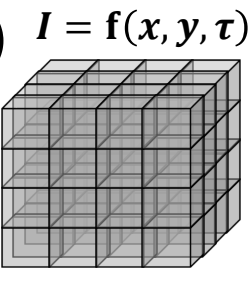

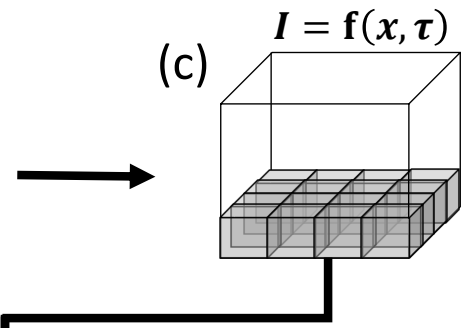
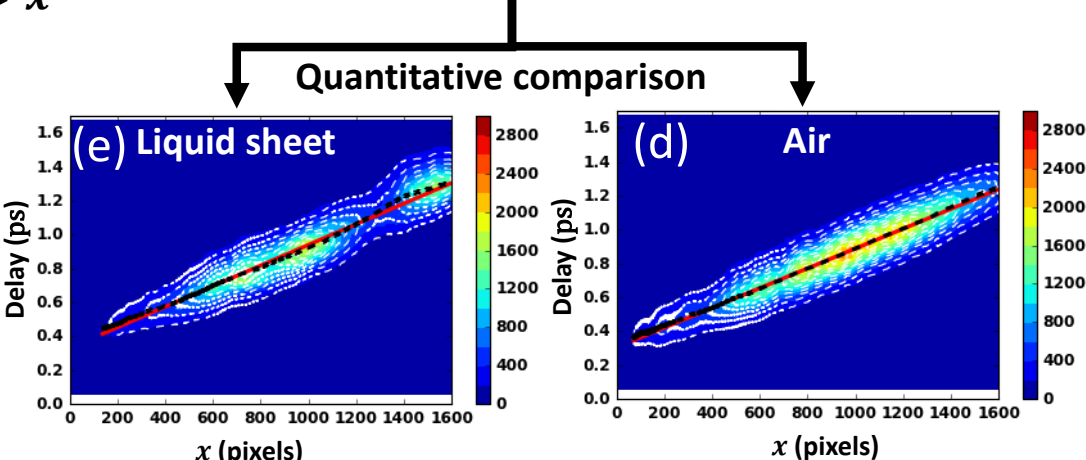

Figure 5. Steps involved in spatio-temporal analysis of time-resolved images

computations are performed using linear regression of maximum locations and gaussian fit modes. At the end, both are linear fits, however, former computes only locations of maximum value of intensity as a reference and latter is based on complete curve fitting of the intensity profile contours to find the location of maximum. The white dashed contours are the gaussian fit modes of intensity w.r.t delay for given $x$ and the color bar represents the measured spatiotemporal evolution of light intensity. The red line is the linear regression of local delay values of maximum intensity for given $x$ and the dashed black curve is the linear regression of the gaussian fit modes. From statistical point of view, we can use linear regression between $x$ and $\tau$, then the linear fit expresses the location of maximum intensity $\left(I_{\max }(x, \tau)\right)$ as a function of $x$ in the form for air :

$$
I_{\max }(\tau)=m I_{\max }(x)+c_{a}
$$

and for liquid sheet,

$$
I_{\max }(\tau)=m I_{\max }(x)+c_{s}
$$

The slope $(m)$ of the spatio-temporal diagram remains constant since it is function of angle $\theta_{n}=9^{\circ}$ between imaging and gating pulse as described in previous section. Now, if we take a difference between intercept of air and liquid sheet, $\Delta \tau=c_{a}-c_{s}$, we obtain an average time delay due to interaction with liquid sheet. For instance, a delay of $\sim 0.135$ picosecond between directly transmitted light through air and transmitted light through liquid sheet is estimated numerically by subtracting the $c_{a}$ and $c_{s}$ of spatio-temporal diagrams $5 \mathrm{~d}$ and $5 \mathrm{e}$. Thus, liquid sheet thickness $(h)$ can be obtain, $h=\frac{\Delta \tau c}{2\left(n_{l}-1\right)}$, where $n_{l}$ is refractive index of liquid.

The computed value for liquid sheet thickness is $\approx 53 \pm 4 \mu \mathrm{m}$ for Figure 2(e). The similar process is applied on a glass plate of known thickness $\left(h_{g}=120 \mu \mathrm{m}\right)$ for calibration purpose. A glass plate under goes delay of $\sim 0.40$ picoseconds and the the computed value of a glass plate thickness is $\approx 117 \pm 3 \mu \mathrm{m}$.

Furthermore, to check variations of liquid-sheet thickness within the field of view $(\sim 10 \mathrm{~mm})$ we carried out the comparison between vertical intensity profile of the images between air and liquid sheet for each particular delay. To do so we divided the $1600 \times 1200$ pixels in rectangular Region of Interest (ROI) of 200 pixel height $(y)$ in the span of 10 intervals. The selection of ROI'S made in such way that each ROI overlaps at the center of prior ROI $(y / 2)$. Next, the 
intensity profile within $\mathrm{ROI}$ is fitted with gaussian profile. As a final step we checked variation of mean and standard deviation (error bars) for gaussian profile for various locations of ROl's as shown in the Figure 5(e). The difference between maximum mean beam location of air and sheet is embedded in small sub-plot for each location of ROl's. This phase shift difference shows that the shift appears roughly constant for different vertical position suggesting that the liquid sheet thickness is nearly constant within the field of view.

\section{Thickness distribution}

We now investigate the variations of the liquid-sheet thickness in the stable regime when one travels downstream from the nozzle exit. Dombrowski et.al [1] carried a theoretical and experimental analysis of the evolution of a liquid-sheet thickness issuing from a flat fan spray nozzle. They proved that the thickness should be inversely proportional to the downstream distance, i.e. $h=K z^{-1}$ where $K$ is a parameter that depends on the nozzle and on the flow regime. As illustrated in Figure 6a, the edges of the liquid sheet are curved and not straight as it would be in the absence of surface tension. However, the sheet can be characterized by an expansion angle at the orifice, namely $2 \alpha_{t}$. This sheet expansion angle and the actual orifice area can be used to derived the expression for thickness parameter $K$.

As shown in Figure 6b, the actual curved area of the flow at the exit of the orifice is represented by $A B J C D I$ of radius of curvature $z_{0}$ and thus thickness parameter can be obtained,

$$
K=A B \cdot z_{0}=\frac{A B \cdot A D}{2 \sin \alpha_{t}}=\frac{\text { Orifice area }}{2 \sin \alpha_{t}}
$$

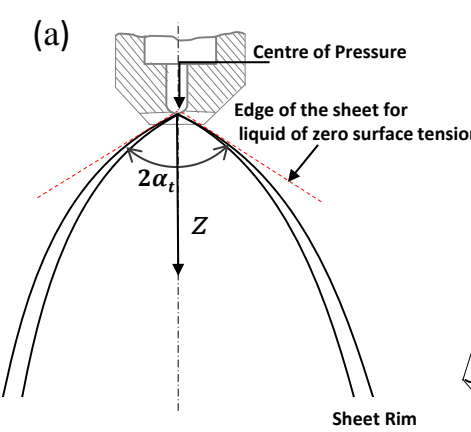

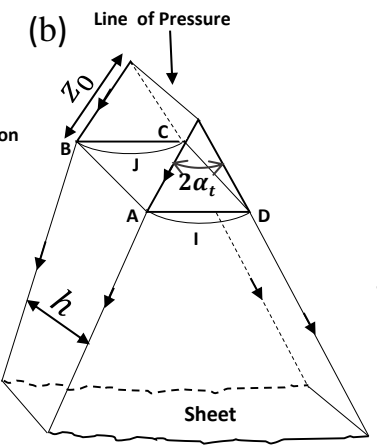

$A B C D=$ Orifice area

$A B J C D I=$ Actual curved area of flow

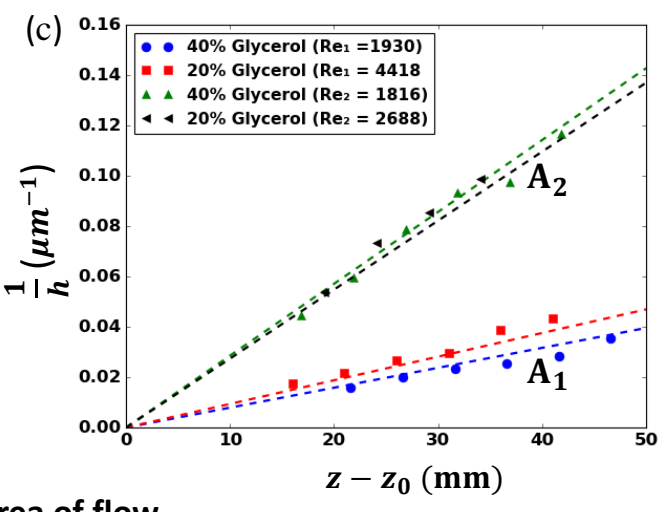

Figure 6. (a)Flow pattern in the sheet(b) flow pattern of liquid upstream and downstream of the orifice [1] (c)Thickness distribution along the centerline of the liquid sheet for nozzle $A_{1}$ and $A_{2}$, dashed line indicates the theoretical lines for different nozzle dimensions and viscosity values

The orifice area based on hydraulic mean diameter for nozzles $A_{1}$ and $A_{2}$ is computed and sheet expansion angle was measured at the nozzle exit for different flow rates. The images acquired from the backlight illumination technique were averaged to obtain included angle between the outer sheet boundaries (Figure 6b). In order to compare theoretical and experimental thickness values, Figure $6 \mathrm{c}$ shows inverse thickness distribution along the sheet centreline for nozzles $A_{1}$ and $A_{2}$ with $40 \%$ and $20 \%$ water-glycerol solution. The typical relationship between $h^{-1}$ and $z-z_{0}$ is obtained by considering the virtual origin $\left(z_{0}\right)$ through which $1 / h$ starts evolving linearly with distance $z$ given by

$$
h=K\left(z-z_{0}\right)^{-1}
$$

A linear relationship should thus exist between $\left(z-z_{0}\right)$ and $1 / h$. Figure $6 \mathrm{c}$ shows an excellent linear correlation between theoretical and experimental values as predicted by equation 5 . The dashed line indicates the theoretical thickness lines. It is noticeable that $1 / h$ does not depend on Reynolds number but only on type of injector $A_{1}$ and $A_{2}$ (at least in the range of parameters studied here). 


\section{Conclusions}

An optical time-gate based on SHG in a BBO crystal allows to obtain the local thickness of stable liquid sheets. It has been shown that an original multi-pass configuration with this gate is able to discriminate delayed light and non-delayed light at the liquid intact region of the nozzle. A spatio-temporal and linear regression analysis is performed to find out space and time relation on statistical series of images with and without liquid sheet. An average time delay due to interaction of light and liquid sheet is then estimated to get the liquid sheet thickness whose evolution with the distance from the nozzle agrees well with Dombrowski's prediction. This optical configuration with large field of view can also yield the enhance contrasted images of the liquid sheet when compared with conventional backlight images. This will be presented in a future work.

\section{References}

[1] Dombrowski, Norman, Hasson, David and Ward, DE. "Some aspects of liquid flow through fan spray nozzles." Chemical Engineering Science Vol. 12 No. 1 (1960): pp. 35-50.

[2] Bush, John W. M. and Hasha, Alexander E. "On the collision of laminar jets: fluid chains and fishbones." Journal of fluid mechanics Vol. 511 (2004): p. 285.

[3] Choo, Y. J. and Kang, B. S. "Parametric study on impinging-jet liquid sheet thickness distribution using an interferometric method." Experiments in fluids Vol. 31 No. 1 (2001): pp. 56-62.

[4] Kondoh, Masato and Tsubouchi, Masaaki. "Liquid-sheet jets for terahertz spectroscopy." Optics express Vol. 22 No. 12 (2014): pp. 14135-14147.

[5] Koralek, Jake D, Kim, Jongjin B et al. "Generation and characterization of ultrathin freeflowing liquid sheets." Nature communications Vol. 9 No. 1 (2018): pp. 1-8.

[6] Idlahcen, Saïd, Rozé, Claude, Méès, Loïc, Girasole, Thierry and Blaisot, Jean-Bernard. "Sub-picosecond ballistic imaging of a liquid jet." Experiments in fluids Vol. 52 No. 2 (2012): pp. 289-298.

[7] Purwar, Harsh, Idlahcen, Saïd, Rozé, Claude, Sedarsky, David and Blaisot, Jean-Bernard. "Collinear, two-color optical Kerr effect shutter for ultrafast time-resolved imaging." Optics express Vol. 22 No. 13 (2014): pp. 15778-15790.

[8] Linne, Mark, Paciaroni, Megan, Hall, Tyler and Parker, Terry. "Ballistic imaging of the near field in a diesel spray." Experiments in fluids Vol. 40 No. 6 (2006): pp. 836-846.

[9] Purwar, Harsh, Idlahcen, Saïd, Rozé, Claude and Blaisot, Jean-Bernard. "Timeresolved imaging with OKE-based time-gate: enhancement in spatial resolution using low-coherence ultra-short illumination." arXiv preprint arXiv:1502.07255 .

[10] Jung, Sungjune, Hoath, Stephen D., Martin, Graham D. and Hutchings, lan M. "Atomization patterns produced by the oblique collision of two Newtonian liquid jets." Physics of Fluids Vol. 22 No. 4 (2010): p. 042101.

[11] Chen, Xiaodong, Ma, Dongjun, Yang, Vigor and Popinet, Stephane. "High-fidelity simulations of impinging jet atomization." Atomization and sprays Vol. 23 No. 12. 